\title{
Defecation as an index of motion sickness in the rat
}

\author{
KLAUS-PETER OSSENKOPP and NANCY LEE FRISKEN \\ University of Western Ontario, London, Ontario, Canada
}

\begin{abstract}
Because defecation in response to body rotation in rats, although noted in a number of previous studies, had not been examined quantitatively, rats in the present two experiments were subjected to a body-rotation-induced taste-aversion procedure, and defecation levels during the body-rotation period were measured. In Experiment 1, rats given saccharin solution followed by $30 \mathrm{~min}$ of body rotation developed a taste aversion and also displayed high levels of defecation during the rotation procedure. Rats given a sham rotation procedure did not develop a saccharin taste aversion and exhibited low levels of defecation. In Experiment 2, rats were first habituated to the rotation apparatus (sham rotation procedure). Subsequent pairing of saccharin solution with body rotation resulted in the development of a taste aversion, and defecation levels during the rotation procedure were dramatically greater than during the habituation period. A control group given sham rotation did not develop a taste aversion and did not increase their defecation levels from the habituation to the conditioning phase. When these control rats were subsequently given body rotation treatments, they exhibited an increase in defecation relative to baseline levels, but with repeated exposures the defecation levels returned to the baseline values. Thus, these animals habituated to the effects of the rotation as indexed by the defecation measure. These results suggest that defecation is a species-relevant index of motion sickness in the rat.
\end{abstract}

Motion sickness is a phenomenon found in a large variety of animal species (Money, 1970). The indicator normally used to quantify motion sickness is the occurrence of an emetic response (Chinn \& Smith, 1955). However, other signs, such as pallor and cold sweating, and symptoms, such as nausea, are frequently associated with this condition in man (Money, 1970). The rat is not capable of an emetic response (Hatcher, 1924), and other criteria are needed to determine whether or not rats experience illness or malaise as a consequence of being subjected to body rotation.

A number of previous studies have used a variety of behavioral measures to index the effects of body rotation in rats. Subjecting rats to body rotation of 8 to 18 rotations per minute (rpm) results in a decrease in spontaneous activity both during and immediately after the rotation procedure (Eskin \& Riccio, 1966). Because reduced activity levels due to body rotation preceded emesis in chimpanzees and squirrel monkeys (Meek, Graybiel, Beischer, \& Riopelle, 1962), it was assumed that the inactivity produced by vestibular stimulation in the rats was attributable to nausea or malaise (Eskin \& Riccio, 1966). Rats

This study was supported by a grant from the Natural Sciences and Engineering Research Council of Canada (U0151) to the first author. Please send reprint requests to the first author at: Department of Psychology, University of Western Ontario, London, Ontario N6A 5C2, Canada. trained on a variety of food-reinforced operant schedules and then subjected to body rotation exhibited systematic response-rate decrements as a function of increasing body rotation rates (Riccio \& Thach, 1968). A postrotational measure indexing the effects of the rotation-elicited unconditioned response (UCR) is suppression of a drinking response in thirsty rats (Haroutunian, Riccio, \& Gans, 1976) given access to water immediately after the rotation period. Another postrotation measure shown to index the effects of body rotation is that of pica; the consumption of a nonnutritive substance (Mitchell, Krusemark, \& Hafner, 1977; Mitchell, Laycock, \& Stephens, 1977). The occurrence of pica also supports the hypothesis that body rotation in nonemetic animals results in gastrointestinal malaise which then elicits pica behavior.

Another method used to index the aversive consequences (UCR) of body rotation in rats is the conditioned-taste-avoidance procedure. Pairing a novel taste, such as saccharin, with the body rotation procedure results in a conditioned avoidance of saccharin with multiple conditioning trials (Green \& Rachlin, 1973; Hutchison, 1973) or even with a single conditioning trial (Braun \& McIntosh, 1973; McCoy, Nallan, \& Pace, 1980). Conditioned taste aversions have been obtained with delays of up to 30 min between access to the novel taste solution and exposure to the body rotation procedure (Haroutunian \& Riccio, 1975). 
One of the signs of motion sickness that sometimes occurs in animals capable of emesis is that of defecation (Money, 1970). For example, dogs often defecate when subjected to the appropriate body motion (Babkin, Dworkin, \& Schachter, 1946). A number of studies examining the effects of body rotation in rats have mentioned the occurrence of defecation as an accompanying response to body rotation (Green \& Rachlin, 1973; Griffith, 1920; Mitchell, Krusemark, \& Hafner, 1977), but no quantitative assessments have been made. The present experiments examined the defecation response in rats subjected to a period of body rotation as another index of motion sickness in these animals. Since rats do not vomit, attempts to characterize the effects of body rotation in these rodents should include as many species-relevant behaviors as possible in order to obtain a detailed picture of motion sickness in this species.

\section{EXPERIMENT 1}

Experiment 1 examined the effects of body rotation on rats' defecation responses during the rotation procedure. These animals were concomitantly subjected to a body-rotation-induced conditionedtaste-aversion procedure to additionally assess the aversive consequences of the body-rotation procedure with this common index.

\section{Method}

Nineteen male hooded rats, weighing 350 to $450 \mathrm{~g}$ at the start of the experiment, were individually housed in stainless steel cages and maintained in a colony room on a 12:12-h light-dark cycle, with lights on from 7:00 a.m. to 7:00 p.m.

The apparatus for producing the rotational stimulation consisted of a turntable capable of revolving at $70 \mathrm{rpm}$ and a $32 \times 32 \times$ $32 \mathrm{~cm}$ Plexiglas chamber mounted on the turntable. The turntable was programmed to rotate for $15-\mathrm{sec}$ periods separated by 5 -sec periods of no rotation. The Plexiglas chamber could be placed on a surface of the apparatus adjacent to the turntable such that a rat in the chamber would be subjected to the noise and vibration of the turntable apparatus but would not be rotated. This condition was designated as a sham rotation condition.

Drinking tubes, calibrated in milliliters and fitted with rubber stoppers and stainless steel drinking spouts containing ball bearings to minimize leakage, were used to measure fluid consumption.

The novel taste solution used in the conditioned-taste-aversion procedure consisted of a solution of sodium saccharin in tap water $(.1 \% \mathrm{w} / \mathrm{v})$ at room temperature $\left(21^{\circ}-23^{\circ} \mathrm{C}\right)$.

Procedure. All rats were adapted to a 23-h/day water-deprivation schedule over a 7-day period. On Days 5 to 7 of the deprivation schedule, water intake by each rat was measured for the 1-h access period by providing two 100 -ml graduated cylinders of tap water (baseline water intake). On Day 8, all rats were given $1 \mathrm{~h}$ of access to the saccharin solution, again presented in two cylinders. Immediately after the $1 \mathrm{~h}$ of access to saccharin, rats from Group ROT $(n=11)$ were individually placed in the Plexiglas chamber on the turntable and rotated at $70 \mathrm{rpm}(15 \mathrm{sec}$ on, $5 \mathrm{sec}$ off) for $30 \mathrm{~min}$. Rats from Group CON $(n=8)$ were placed in the Plexiglas chamber and exposed to the sham rotation condition for $30 \mathrm{~min}$. On Day 9, all rats were presented with a two-bottle choice test (Dragoin, McCleary, \& McCleary, 1971; Grote \& Brown,
1971) for $1 \mathrm{~h}$; one bottle contained tap water, the other bottle saccharin solution. Two more blocks of a conditioning trial (Group ROT) or control trial (Group CON) followed by a two-bottle choice-test trial were given over the next 4 days. On Day 14, the rats received access to the saccharin solution for $1 \mathrm{~h}$.

When rats were given the two-bottle choice tests, the positions of the water bottle and saccharin bottle were randomly varied over test days to avoid the formation of a position habit. The dependent measures in this experiment consisted of (1) recording at the end of each 30-min body-rotation or sham body-rotation period the number of fecal boluses deposited by the rat in the Plexiglas chamber, (2) measuring the amount of saccharin solution consumed on each of the conditioning days, and (3) calculating a saccharin preference ratio (total saccharin intake divided by total fluid intake) on each of the test days.

\section{Results}

The group mean data from the first experiment are presented in Figure 1. Inspection of this figure shows that Group CON increased saccharin intake on conditioning days, whereas Group ROT exhibited a relative decrease in saccharin consumption. Analysis of variance for repeated measures (conditioning days) revealed a highly significant group $\times$ days interaction $[F(3,51)=11.78, p<.001]$, and simple main effects tests (Kirk, 1968) indicated significant group differences on the $3 \mathrm{rd}$ and 4 th conditioning days (ps < .05). Comparison of the group mean saccharin preference measure in Figure 1 clearly shows an increase in preference over days for the control group (Group CON) but a decrease in preference for the experimental group (Group ROT). Statistical analysis again supported this comparison: a significant group main effect $[F(1,17)=73.12, p<.001]$ and a significant group $\times$ days interaction $[F(2,34)=$ $7.85, p<.002$ ]. Post hoc simple effects tests in-

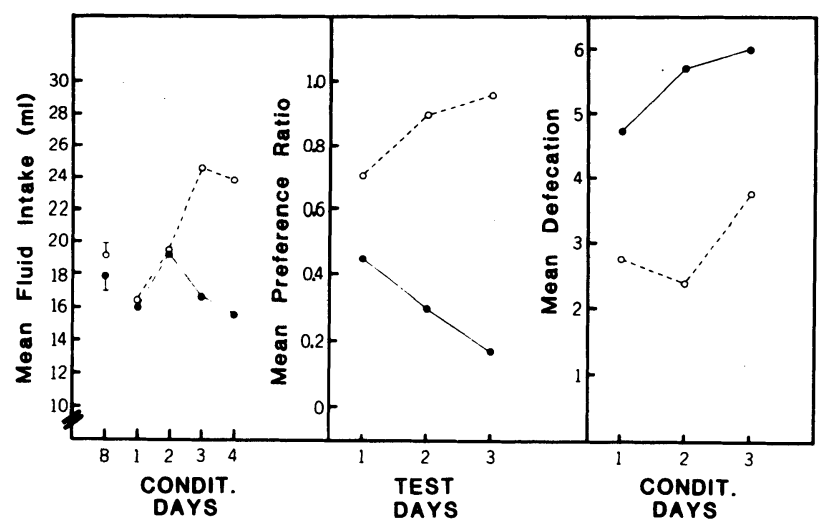

Figure 1. Left panel: Mean fluid intake for the control and experimental groups during the baseline (B) phase (water intake) and on the $\mathbf{4}$ conditioning days (saccharin intake). Middle panel: Mean saccharin preference ratios for the control and experimental groups as a function of test days. Right panel: Mean defecation levels in the control and experimental groups during the sham rotation and rotation procedure, respectively, on the 3 conditioning days. Control group (Group CON)-open circles; experimental group (Group ROT)-filled circles. 
dicated group differences in the preference measure on all 3 test days (ps $<.05)$.

The defecation measure also showed substantial group differences over test days. Group ROT defecated significantly more than Group $\mathrm{CON}$ over conditioning days [significant group main effect, $F(1,17)=$ $5.56, \mathrm{p}<.05$ ], indicating that the rotation procedure significantly increased the number of defecation responses. Product-moment correlations between mean saccharin preference ratios (Test Days 1-3) and mean defecation levels (Conditioning Days 1-3) were obtained for each group. Group ROT showed a correlation of .24 and Group CON a correlation of .05 between these two measures. Neither of these correlations was statistically significant. Nonsystematic observation also indicated that the defecation responses were distributed over the 30-min rotation period and did not occur only at the initiation of the rotation procedure.

\section{Discussion}

Consistent with a number of previous studies (Braun \& McIntosh, 1973; Green \& Rachlin, 1973; Hutchison, 1973), body rotation was found to be effective in producing a saccharin taste aversion when paired with this taste stimulus. This taste aversion was evident in both the saccharin preference data on test days and in the saccharin intake data on conditioning days. In addition to developing an aversion to the saccharin taste stimulus, rats subjected to the body rotation procedure also exhibited significantly more defecation responses to the body-rotation procedure than did the control group. Thus, defecation levels also discriminated between the experimental and control groups. Although a significant correlation between degree of taste aversion and level of defecation in response to rotation was not found, this does not invalidate the use of defecation as an index of motion sickness. Many other factors, such as level of emotionality, level of conditionability, etc., probably obscure any simple relationship one might expect to find between the two measures of motion sickness used in this experiment.

The observation that defecation responses were distributed across the 30 -min rotation period suggests that the defecation responses were probably not elicited by the novelty of the rotational stimulation but, rather, represented the effect of some other aversive state, such as nausea. However, systematic data are needed to clarify this point. Taken together with the taste-aversion results, the present measure of defecation suggests that it is a useful index of motion sickness in rats.

\section{EXPERIMENT 2A}

Experiment 1 used a between-groups comparison to show that defecation levels increase when rats are subjected to body rotation. In Experiment 2, we used an experimental design that allowed for both a between-groups and a within-group comparison to further examine the effects of body rotation on defecation. Exposing rats to a novel environment tends to elicit defecation responses often interpreted as reflecting the animals' level of emotionality (Hall, 1934). Since the Plexiglas holding chamber was a novel environment on the first conditioning day in Experiment 1, it could be argued that part of the defecation responses resulted from emotional reactivity to the novel environment rather than from the effects of the body rotation. In Experiment $2 \mathrm{~A}$, rats were first familiarized to the holding chamber and the accompanying noise and vibration and then subjected to the body-rotation or sham rotation procedures. Thus, Experiment 2A was designed to assess possible interactions between the effects of the novelty of the rotation chamber and the effects of the rotational stimulation. By eliminating the novel qualities of the apparatus, only the effects of rotational stimulation per se would be evident.

\section{Method}

Twenty male hooded rats, weighing 300 to $400 \mathrm{~g}$ at the start of the experiment, were housed as in Experiment 1. The apparatus used in Experiment 1 was also used in Experiment 2A, and the same novel taste solution (.1\% saccharin) was also used.

Procedure. All rats were adapted to a 23-h/day water-deprivation schedule over a 7-day period. On Days 5 to 7 of the deprivation schedule, water intake by each rat for the 1-h access period was measured by providing two 100 -ml cylinders of tap water (baseline water intake). On Days 8, 10, and 12, all rats were placed in the Plexiglas chamber and exposed to the noise and vibration of the rotation apparatus but were not rotated (sham rotation procedure). Water intake during the 1-h access period was again measured on these days (habituation water intake). On Day 13, Group ROT $(n=12)$ was given 1-h of access to the saccharin solution, and then each rat was placed in the rotation chamber and rotated at $70 \mathrm{rpm}(15 \mathrm{sec}$ on, $5 \mathrm{sec}$ off) for $30 \mathrm{~min}$. Group CON $(n=8)$ was given the saccharin solution and then subjected to $30 \mathrm{~min}$ of the sham rotation procedure. On Day 14, all rats were given a two-bottle saccharin preference test, as in Experiment 1. Days 15 and 17 constituted 2 more conditioning days, and Days 16 and 18, 2 more test days. The same dependent measures as in Experiment 1 were recorded in this experiment.

\section{Results}

Figure 2 depicts the group mean results over days on the three dependent measures.

Analysis of variance on the baseline and habituation water-intake levels indicated no significant effect of group condition or phase of experiment. However, saccharin intake over the 3 conditioning days differed significantly between the two groups [significant group $\times$ days interaction, $F(2,36)=9.46$, $\mathrm{p}<.001$ ]. Post hoc simple main effects tests (Kirk, 1968) indicated a significantly lower intake for Group ROT on Conditioning Day 3 only. Analysis of the saccharin preference data also revealed a significant group $\times$ days interaction $[F(2,36)=6.44$, $\mathrm{p}<.005$ ], with post hoc tests indicating a significantly lower preference for Group ROT on Test 


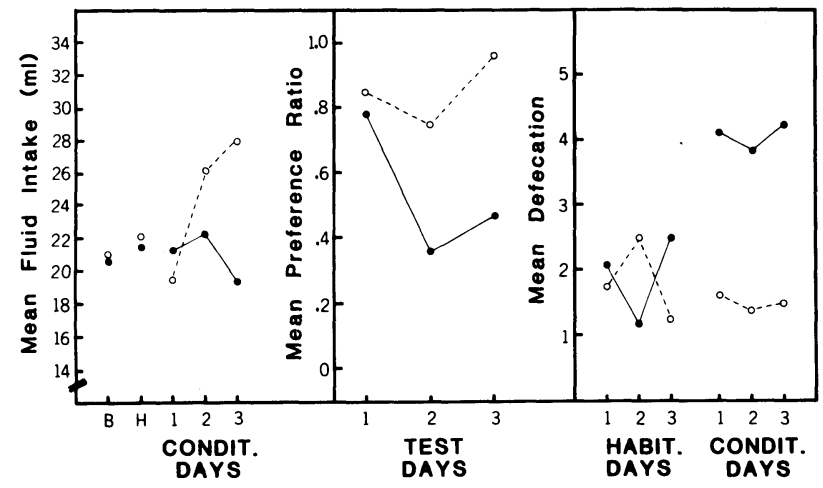

Figure 2. Left panel: Mean fluid intake for the control and experimental groups during the baseline (B) phase, the habituation (H) phase (water intake for both $B$ and $H$ ), and on the 3 conditioning days (saccharin intake). Middle panel: Mean saccharin preference ratios for the control and experimental groups as a function of test days. Right panel: Mean defecation levels in the control and experimental groups during the habituation phase and the conditioning phase. Control group (Group CON)-open circles: experimental group (Group ROT)-filled circles.

Days 2 and 3. Thus, both the saccharin intake data and the saccharin preference data revealed a conditioned taste aversion to saccharin in the experimental group.

Analysis of the defecation data indicated a highly significant group $\times$ condition interaction $[F(1,18)=$ $15.90, \mathrm{p}<.001]$ produced by greater levels of defecation on conditioning days in Group ROT relative to the control group and no group differences during habituation (see Figure 2). Inspection of these data shows an increase in defecation in Group ROT when they were subjected to rotation, whereas Group CON had similar levels of defecation in both the habituation and the conditioning phase. These results show that a within-group comparison also demonstrates a clear effect of body rotation on defecation levels in rats. Again, product-moment correlations between mean saccharin preference ratios (Test Days 1-3) and mean defecation levels during conditioning (Conditioning Days 1-3) were obtained for both groups. Group ROT showed a correlation of .67 ( $\mathrm{p}<.02)$, whereas Group CON showed a correlation of $-.06(p>.05)$. Correlations between mean saccharin preference ratios and mean defecation levels during the habituation phase were also obtained. Group ROT again showed a moderate but nonsignificant positive correlation $(r=.48, p>.05)$, and Group CON showed a small negative correlation $(r=-.21, p>.05)$. Nonsystematic observation again suggested that defecation responses were distributed across the 30-min rotation period.

\section{Discussion}

In Experiment 2A, the rats were first habituated to the chamber in which they were subjected to body rotation. Thus, the situational variables, other than, of course, the rotation itself, were not novel to the rats during the first rotation session. Presumably, the increased levels of defecation seen in the rats subjected to rotation (a $112 \%$ increase) was produced mainly by the adverse consequences of the rotational stimulation rather than by emotional reactivity to the situational variables. The findings of Experiment $2 \mathrm{~A}$ suggest that the rotation environment does not need to be novel to produce either a taste aversion or an increase in defecation levels and that a within-group comparison is quite adequate for demonstration of increased defecation levels. The finding that mean saccharin preference ratios correlate positively with mean defecation level during rotation might, at first glance, suggest that one or the other index of motion sickness used in the present experiment is not valid. A negative correlation would be expected if a simple relationship between degree of taste aversion and amount of defecation in response to rotation existed. That is, one might argue that increased nausea produced by body rotation should increase the degree of taste aversion observed and also increase the amount of defecation in response to the nausea. However, other factors, such as degree of conditionability of the animal or basal levels of emotionality, might obscure or even reverse the expected relationship. The finding that defecation levels during habituation, presumably a measure of emotional reactivity, also show a positive correlation with mean saccharin preference ratios tend to support this argument. Less emotional animals tend to show a greater level of conditioned taste aversions in response to body rotation. The relationships between degree of body-rotation-induced conditioned taste aversion and levels of other indices of motion sickness clearly need further examination.

\section{EXPERIMENT 2B}

One of the common characteristics of motion sickness seems to be a decrease in the severity of the signs and symptoms with repeated exposure to motion (Tyler \& Bard, 1949). This habituation effect seems to be quite apparent in humans, whereas dogs seem to be relatively resistant (Noble, 1948; Schwab, 1954). There is also evidence to suggest that rats show habituation to repeated vestibular stimulation. Decreases in postrotation nystagmus (Griffith, 1920) and in amount of postrotational hypoactivity (Riccio, Igarashi, \& Eskin, 1967) have been noted with repeated exposure to the vestibular stimulation. Pica induced by vestibular stimulation also shows the habituation effect with repeated exposures (Mitchell, Krusemark, \& Hafner, 1977). In Experiment 2B, we examined the effects of repeated exposure to body rotation on defecation levels.

\section{Method}

The eight control rats from Experiment $2 \mathrm{~A}$ were used in Experiment $2 \mathrm{~B}$. These rats were placed on ad-lib food and water 
and housed as in Experiment 1. The rotation apparatus used in Experiment 1 was also used in Experiment 2B.

Procedure. Experiment 2B commenced 7 days after the conclusion of Experiment 2A. On Days 1-3 (baseline), all rats were placed in the Plexiglas chamber and exposed to the noise and vibration of the rotation apparatus for $30 \mathrm{~min}$ (sham rotation procedure). On Days 4-11 (treatment), all rats were placed in the rotation chamber and rotated at $70 \mathrm{rpm}(15 \mathrm{sec}$ on, $5 \mathrm{sec}$ off for $30 \mathrm{~min}$. The dependent measure recorded was the number of fecal boluses deposited in the chamber during the 30 -min sham rotation or body-rotation periods.

\section{Results}

Mean defecation levels as a function of days are presented in Figure 3. Inspection of this figure indicates an increase in defecation, relative to baseline, when the rats were subjected to the body-rotation procedure. With repeated exposures to the rotation procedure, the defecation levels return to baseline values (last 2 days). A subject $\times$ treatment analysis of variance of the baseline and first 3 days of the treatment data indicated a significant increase in defecation level from baseline to initial treatment phase $[F(1,35)=7.45, p<.01]$. Thus, defecation levels increased significantly above baseline values during initial rotation treatments but returned to baseline values after six experimental sessions (habituation effect).

\section{Discussion}

The results of Experiment 2B present evidence of habituation of the effects of vestibular stimulation in rats when indexed by defecation levels. Thus, consistent with previous research on rats in which other indices of vestibular stimulation were examined (Griffith, 1920; Riccio et al., 1967), defecation levels also reflect the habituation effect of motion sickness with repeated exposure. Experiment $2 B$ also partially replicates the findings of Experiment $2 \mathrm{~A}$ by demonstrating an increase in defecation levels in rats subjected to body rotation (again, by using a withinsubjects design).

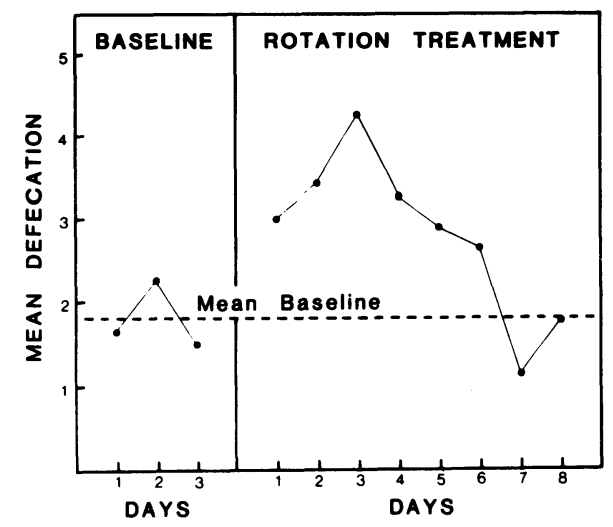

Figure 3. Mean defecation levels during the habituation phase (baseline) and the treatment phase (daily exposure to body rotation).

\section{GENERAL DISCUSSION}

The present two experiments provide a quantitative demonstration of increased defecation responses in rats subjected to body rotation and of habituation of this response with repeated vestibular stimulation. Taken together with the demonstrations of body-rotation-induced taste aversions in the same animals, these results suggest that defecation level is another variable that can be used to index the effects of rotational stimulation in rats. Mitchell, Krusemark, and Hafner (1977) used informal observation of defecation responses in their rats subjected to body rotation to support the presence of gastrointestinal malaise produced by the rotation procedures. These authors have also argued that it is desirable to find physiologically relevant behaviors (such as emesis, anorexia, diarrhea, or defecation) to index motion sickness if a comparative analysis of motion sickness and the psychophysics of gastrointestinal malaise is to be carried out. The present experiments now add defecation level as another species-relevant index of motion sickness in the rat. Thus, it is now possible to assess the presence of motion sickness in rats by using several purely observational techniques (such as measuring hypokinesia, pica, and defecation levels), rather than by relying on manipulation of the animals' behavior with conditioning procedures (such as conditioned taste aversions or decreased operant response levels) to index the effects of rotational stimulation.

\section{REFERENCES}

Babkin, B. P., Dworkin, S., \& Schachter, M. Experimental motion sickness and attempts at therapy. Revue Canadienne de Biologie, 1946, 5, 72-86.

Braun, J. J., \& McIntosh, H. Learned taste aversion induced by rotational stimulation. Physiological Psychology, 1973, 1, 301-304.

Chinn, H. I., \& Smith, P. K. Motion sickness. Pharmacological Reviews, 1955, 7, 33-82.

Dragoin, W., McCleary, G. E., \& McCleary, P. A comparison of two methods of measuring conditioned taste aversions. Behavior Research Methods \& Instrumentation, 1971, 3, 309-310.

Eskin, A., \& Riccio, D. C. The effects of vestibular stimulation on spontaneous activity in the rat. Psychological Record, 1966, 16, 523-527.

Green, L., \& RAchlin, H. The effect of rotation on the learning of taste aversions. Bulletin of the Psychonomic Society, 1973, 1, 137-138.

Griffith, C. R. The effect upon the white rat of continued bodily rotation. American Naturalist, 1920, 54, 524-534.

Grote, F. W., \& Brown, R. T. Conditioned taste aversions: Two-stimulus tests are more sensitive than one-stimulus tests. Behavior Research Methods \& Instrumentation, 1971, 3, 311-312.

HALL, C. S. Emotional behavior in the rat: Defecation and urina- 
tion as measures of individual differences in emotionality. Journal of Comparative Psychology, 1934, 18, 385-403.

Haroutunian, V., \& Riccio, D. C. Acquisition of rotationinduced taste aversion as a function of drinking-treatment delay. Physiological Psychology, 1975, 3, 273-277.

Haroutunian, V., Riccio, D. C., \& Gans, D. P. Suppression of drinking following rotational stimulation as an index of motion sickness in the rat. Physiological Psychology, 1976, 4, 467-472.

Hatcher, R. A. The mechanism of vomiting. Physiological Reviews, 1924, 4, 479-504.

Hutchison, S. L. Taste aversion in albino rats using centrifugal spin as an unconditioned stimulus. Psychological Reports, 1973, 33, 467-470.

KIRK, R. E. Experimental design: Procedures for the behavioral sciences. Belmont, Calif: Brooks/Cole, 1968.

McCoy, D. F., Nallan, G. B., \& Pace, G. M. Some effects of rotation and centrifugally produced high gravity on taste aversion in rats. Bulletin of the Psychonomic Society, 1980, 16, 255-257.

Meek, J. C., Graybiel, A., Beischer, D. E., \& Riopelle, A. J. Observations of canal sickness and adaptation in chimpanzees and squirrel monkeys in a "slow rotation room." Aerospace Medicine, 1962, 33, 571-578.
Mitchell, D., Krusemark, M. L., \& Hafner, E. Pica: A species relevant behavioral assay of motion sickness in the rat. Physiology \& Behavior, 1977, 18, 125-130.

Mitchell, D., Laycock, J. D., \& Stephens, W. F. Motion sickness-induced pica in the rat. American Journal of Clinical Nutrition, 1977, 30, 147-150.

Money, K. E. Motion sickness. Physiological Reviews, 1970, 50, 1-39.

NoBle, R. L. Adaptation to experimental motion sickness in dogs. American Journal of Physiology, 1948, 154, 443-450.

Riccio, D. C., Igarashi, M., \& Eskin, A. Modification of vestibular sensitivity in the rat. Annals of Otology, Rhinology and Laryngology, 1967, 76, 179-188.

Riccio, D. C., \& ThACH, J. S. Response suppression produced by vestibular stimulation in the rat. Journal of the Experimental Analysis of Behavior, 1968, 11, 479-488.

SchwaB, R. S. The nonlabyrinthine causes of motion sickness. International Record of Medicine, 1954, 167, 631-637.

Tyler, D. B., \& BARD, P. Motion sickness. Physiological Reviews, 1949, 29, 311-361.

(Manuscript received April 9, 1982;

revision accepted for publication August 2, 1982.) 